\title{
Development of Categorizing Ability in Preschoolers
}

\author{
Xiaoxi Wang ${ }^{1}$, Zhicheng Zhang ${ }^{1}$, $\mathrm{Na} \mathrm{Hu}^{1, a *}$ \\ ${ }^{1}$ Department of preschool education andspecial education, Kunming University, Kunming China \\ ${ }^{a}$ Email: hunoo77@126.com
}

\begin{abstract}
Categorical thinking is the underlying capability of information technology. In order to get more understand the development of children categorization ability, two experiments were employed in this research. 60 children (30 boys, 30 girls) joined the experiment, there are 20 children for each age group(3-4 years, 4-5 years, 5-6 years). In experiment 1 children were asked to categorize the pictures freely; in experiment 2 children were asked to categorize based on the given example.The result showed that most 5-6 years old children categorize based on taxonomic rules, while younger children more categorize based on similarity and thematic relationship. This result showed that children's thinking developed form surface similarity to essential connection. The way of the categorization (free/forced) more influenced the 3-4 years children, in the free categorization $10 \%$ of them can't categorize the picture, but all the children can do the categorization in the forced task. This result showed that younger children's thinking flexibility was not fully developed, they need a clearly introduction to help. Based on the results above, education suggestions were given that children be trained based on their age characteristics and critical period should be grasped in thinking development, so that can help develop their information technology literacy.
\end{abstract}

Keywords: Category Ability Development, Kindergarten Children; Education Suggestions; Information Technology

\section{INTRODUCTION}

People can distinguish between different things and react differently by categorization processing .Categorization is the base and core ability of human cognition. As a working definition, categories are groups of distinct abstract or concrete items that the cognitive system treats as equivalent for some purpose[1].Categorization ability developed from young age, children natural categories are acquired in their daily life or interacting with the categories and with category members. Such as their observation of fruit, they get understand the category of the colour, shape, taste . But while the category learning, there are different standard in their category, such as based on the surface similarity (e.g. the same colour); thematic relationship (e.g. milk and bread) and taxonomic relationship (e.g. apple is a kind of fruit). Thematic categories involve heterogeneous members belonging to a common event or scene, and thus organized in spatial and /or temporal relations of contiguity[2]. In taxonomic categories, elements are linked together because they share common properties; they are the "same sort" of thing[3].

Classification ability developed from very young age,
Ross used the habituation method to study the classification ability of infants aged 6 to 10 months. In the experiment, the researchers repeatedly presented infants with certain categories of objects (such as a man's face or a toy animal) until the baby was no longer interested and the gaze time began to decrease. Once the infant is habituated to this type of object, it is presented with a new type of sting (e.g. a woman's face or another toy animal). It is found that in this case, the infant's fixation time to the new type of stimulus is significantly increased. This result suggests that even 6-month-olds have the ability to form categories[4].

The 1930s Vygotsky began to study children's classification ability. In the study, Vygotsky used wood blocks of different shapes, sizes and colors as stimulus materials and placed them together in front of children to divide them into several piles. Through this research, Vygotsky found that the development of children's classification ability can be divided into the following stages: the stage of subjective impression, the stage of temporary rules and the stage of determining rules. In the stage of subjective impression, children classify wood blocks according to their own subjective wishes without any classification rules; in the temporary rule stage, children classify wood blocks by color at some 
time, and sometimes by shape, and the classification criteria vary from time to time; in the fixed standard stage, children can classify all the stimuli according to a fixed standard[5] 。

But while more and more attention paid on children categorization development, results showed that even young children showed categorization flexibility. Flexible categorization involves the ability to categorize an object on one basis (e.g. Taxonomically) on one occasion, and on another basis (e.g. thematically) on another occasion[6].Study of 3-5 year old children showed that even as young as 5 year old, they showed flexibility between thematic and taxonomic relationship.[7]

in order to gain more understanding of development of children categorization ability and categorization flexibility, we employed kindergarten children form 3-6years as participants, use free categorization and forced categorization task to explore how the age and the way of categorization ways affect the categorization processing.

\section{EXPERIMENT1 CATEGORIZATION OF 2D MATERIAL}

\subsection{Participants}

A total of 60 children, 30 boys and 30 girls, were randomly selected from $\mathrm{R}$ kindergarten in Kunming City, China. The age range of the children divided into three groups: 3-4 years old (20children,10male and 10female), 4-5 years old (20children,10male and 10female)), and the large class is 5-6 years old (20children,10male and 10female).

\subsection{Material}

16 pictures of four category (animal, plant, vehicle and stationery) were employed into the experiment.

\subsection{Procedure:}

Before the experiment, teacher told the children the name of each picture, and test each child to make sure they get a clearly understand of each picture.

\subsubsection{Sub-expriment 1:Free categorization}

1. Setting the situation: "today, mother rabbit asked the baby rabbit to help arrange the toy pictures at home, but there are too many pictures, so I would like to ask you to help the baby rabbit to sort out the pictures together, OK?"
2. Show the scrambled pictures and let the children know the content of each picture.

3 . Let the children classify 16 pictures freely.

4. Instructions: children, please put these pictures on the table together.

5. Observe and record the classification process and results of children, ask and record the reasons for classification.

\subsubsection{Sub-expriment 2:forced categorization}

1. Setting the situation: "the mother rabbit gave the baby rabbit four boxes, put the pictures of banana, football, bicycle and fish into four boxes, and asked the rabbit to divide the pictures into four categories."

2. Instructions:Boys and girls, please help the little rabbit send the rest of the picture on the table back to the box you think belongs to it.

3 . Let the children classify the 16 pictures according to the target image.

4. Observe and record the classification process and results of children, ask and record the reasons for classification.

\subsection{Result}

\subsubsection{Result of free categorization}

After the statistics of the classification results of the subjects, the classification criteria proposed by the subjects were divided into four levels.

First, it cannot categorize: In the process of categorization, they showed no purpose, no order, random classification,;after the categorization, pictures have no correlation, and cannot give reasons.

Secondly, categorization based on the surface similarity of pictures. According to the color, size, shape and other external characteristics of real pictures, they can be divided into one category.

Thirdly, categorization based on thematic relationship. Two or more physical pictures often appear in the same situation or can be divided into one category according to their functions.

Fourth, categorization based on taxonomic relationship. It can be divided into one category according to the category or essential connection of the physical pictures.

The detailed result showed below: 
Table 1. Result of free categorization

\begin{tabular}{|c|c|c|c|c|c|}
\hline Age & $\begin{array}{c}\text { Can't } \\
\text { categorize }\end{array}$ & $\begin{array}{c}\text { Categorize on } \\
\text { similarity }\end{array}$ & $\begin{array}{c}\text { Categorize on } \\
\text { thematic } \\
\text { relationship }\end{array}$ & $\begin{array}{c}\text { Categorize on } \\
\text { taxonomic } \\
\text { relationship }\end{array}$ & total \\
\hline $3-4$ & $2(10 \%)$ & $4(20 \%)$ & $9(45 \%)$ & $5(25 \%)$ & $20(100 \%)$ \\
\hline $4-5$ & $0(0 \%)$ & $6(30 \%)$ & $9(45 \%)$ & $5(25 \%)$ & $20(100 \%)$ \\
\hline $5-6$ & $0(0 \%)$ & $4(20 \%)$ & $5(25 \%)$ & $11(55 \%)$ & $20(100 \%)$ \\
\hline
\end{tabular}

On the free categorization task, no child cannot categorize the pictures, 3-4 age group and 4-5 age group showed similar tendency, $45 \%$ of children categorize on thematic relationship, for the 4-5age group there are $30 \%$ of them categorize based on similarity, while there are $20 \%$ in 3-4 years group; for these both groups, there are $5 \%$ of each group categorized based on taxonomic relationship. There are $10 \%$ of 3-4year group, they can;t

categorize the pictures.

For the 5-6 group, most of them (55\%) categorize based on the taxonomic relationship, $25 \%$ categorize based on thematic relationship, $20 \%$ of them categorize based on similarity.

\subsubsection{Result of forced categorization}

Table 2. Result of forced categorization

\begin{tabular}{|l|l|l|l|l|l|}
\hline Age & $\begin{array}{l}\text { Can't } \\
\text { categorize }\end{array}$ & $\begin{array}{l}\text { Categorize on } \\
\text { similarity }\end{array}$ & $\begin{array}{l}\text { Categorize on } \\
\text { thematic } \\
\text { relationship }\end{array}$ & $\begin{array}{l}\text { Categorize on } \\
\text { taxonomic } \\
\text { relationship }\end{array}$ & total \\
\hline $3-4$ & $0(0 \%)$ & $14(70 \%)$ & $2(10 \%)$ & $4(20 \%)$ & $20(100 \%)$ \\
\hline $4-5$ & $0(0 \%)$ & $7(35 \%)$ & $8(40 \%)$ & $5(25 \%)$ & $20(100 \%)$ \\
\hline $5-6$ & $0(0 \%)$ & $3(15 \%)$ & $4(20 \%)$ & $13(65 \%)$ & $20(100 \%)$ \\
\hline
\end{tabular}

In the forced categorization task, all children can do the categorization, for the 3-4years age group, $70 \%$ of them categorize based on the similarity, $10 \%$ based on thematic relationship, $20 \%$ based on taxonomic relationship. For $4-5$ years age group, $35 \%$ of them based on similarity, $40 \%$ based on thematic relationship; $25 \%$ based on taxonomic relationship. For 5-6 years age group, only $15 \%$ of them categorize based on similarity, $20 \%$ of them categorize based on thematic relationship, most of them (65\%)categorize base on taxonomic relationship.

\section{DISSCUSSION}

The development of classification standards basically conforms to the development law of from surface similarity to taxonomic relationship. Younger children like to categorize based on the similarity, especially in the forced categorization task $70 \%$ of 3-4 age group categorize based on the similarity, while there are 65\% 5-6 group can categorize based on taxonomic relationship. Taxonomic relationship represented the more complex and deep thinking of the relationships. Thematic relationship is a kind of relationship that accord to children's daily life, such as cow and milk which can be find easily in their daily life, so a certain percentage of children categorize based on thematic relationship. In the free categorization task, for both 3-4 and 4-5 years group, $45 \%$ of children categorize based on the thematic relationship, while in the forced categorization task $45 \%$ of $4-5$ years children categorize based on the thematic relationship. Based on all the results, it is clearly showed that 5 years old may be a critical period of thinking development.

Free or forced way influenced children's categorization task. In the free categorization task, $10 \%$ of 3-4 years group children can't do the categorization task, but all the children can do the categorization task in the forced task. This result may showed that child need a clearly introduction, in the free task, they were only asked to put pictures together, but in the forced task, a sample was given, the introduction was more clear, children can do the categorization based on the sample. 3-4 years old children showed the biggest difference between this two ways of categorization. This may caused by that younger children thinking based one the representation, in the forced categorization task, a 
sample of each category was given, their judgement was influenced by the visual images of the sample, they may categorize based on" how similar it looks like the sample". But in the free categorization task, young child don't have a certain sample, so they may use another strategy to categorize, such as their daily experience, this induction can explain why there are $45 \%$ of $3-4$ years children categorize based on thematic relationship in the free categorization task.

\section{CONCLUSION}

In conclusion, in all three age groups,children showed better in forced categorization . There is a target in the forced categorization, when classified according to the target, the target is used as a representative of the generic relationship. When children select objects with similar characteristics from other physical images, the target is like a fixed reference. Children can directly compare the similarities between the target and the selected objects. Children with low classification ability can classify them through external feature selection, Those with high classification ability can be classified according to the theme relationship, function relationship and even generic relationship of physical pictures through association or reflection of life experience.

In this study, 3-4-year-old children take surface similarity as the main classification standard, 5-6-year-old children take generic relationship as the main classification standard, while 4-5-year-old children take thematic relationship as the classification standard, but there is little difference from surface similarity and generic relationship as the classification standard. In general, the development of classification standards for 3-year-old children from small class to 6-year-old basically conforms to the trend from surface similarity to theme (function) relationship and then to generic relationship.

\section{ACKNOWLEDGMENTS}

This paper was supported by the research multi-cultural curriculum for kindergartens in Ethnic areas of Yunnan Province.

\section{REFRENCES}

[1]. Markman, A.B. and B.H. Ross, Category use and category learning. Psychological Bulletin, 2003. 129(4): p. 592-613.

[2]. Murphy, G.L., Causes of taxonomic sorting by adults: A test of the thematic-to-taxonomic shift. Psychonomic Bulletin \& Review, 2001. 8(4): p. 834-839.

[3]. Mandler, J.M., On concepts. Cognitive Development, 1993. 8(2): p. 141-148.

[4]. Ross, G.S., Categorization in 1-to 2-year-olds. 1980. p. 391-396.

[5]. Vygotsky, L.S., Thought and language. Bulletin of the Orton Society, 1964. 14(1): p. 97-98.

[6]. Deák, G. and P.J. Bauer, The effects of task comprehension on preschoolers' and adults' categorization choices. J Exp Child Psychol, 1995. 60(3): p. 393-427.

[7]. Blaye, A. and F. Bonthoux, Thematic and taxonomic relations in preschoolers: The development of flexibility in categorization choices. British Journal of Developmental Psychology, 2011. 19(3): p. 395-412. 